\title{
Ein bedeutsames Quartärprofil in einer Höhlenruine bei Hunas/Hartmannshof (Nördliche Frankenalb)
}

\author{
2. Vorbericht
}

\author{
Von Florian Heller, Erlangen-Nürnberg ')
}

$\mathrm{Zusammenf}$ assung: Zum ersten Mal wird von authentischer Seite ausführlicher über die Ergebnisse einer seit 7 Jahren im Gebiete der nördlichen Frankenalb durchgeführten paläontologisch-urgeschichtlichen Grabung berichtet. Das in einer Höhlenruine bei Hunas (ca. $40 \mathrm{~km}$ östlich von Nürnberg) erschlossene vieıgliederige Schichtenprofil reicht vom Würm über das Riß-WürmInterglazial bis in das Riß zurück. Diese Datierung kann sich nicht allein auf eine reiche Ausbeute an Saugetierresten stützen, sondern findet ihre weitere Untermauerung auch durch die petrographisch-pedologische Untersuchung des Sedimentmaterials. Besondere Bedeutung erlangt der Fundplatz schließlich durch die Entdeckung von insgesamt über 250 ortsfremden Silices, die aus nicht weniger als 5 verschiedenen Niveaus des Riß-Würm und des Riß stammen.

$\mathrm{Summary}:$ For the first time here is given a more detailed authentic report on the resuits of a 7 year series of paleontological-prehistorical excavations in the northern Frankenalb area. In the ruins of an old cave near Hunas (ca. $40 \mathrm{kms}$ east of Nürnberg) a sealed-off profile containing numerous strata was found, which covers a period of time from the Würm, through the Riß-WürmInterglacial to the Riß ice-age. This determination of the age cannot only be based on the rich mass of mammalian-remains, but also is strengthened by the petrographical-pedological examination of the sediments. Lastly the site obtained a place of special importance by the discovery of a total of more than 250 allochthonous silices, which were recovered trom altogether five different Riß-Würm and Riß levels.

Ohne Wissen und vor allem gegen den Willen des Verfassers dieser Zeilen erschienen Ende Juni/ Anfang Juli 1962, von völlig unberufener Hand geschrieben, in der örtlichen Tagespresse Notizen über "sensationelle Höhlenfunde“ im Landkreis Hersbruck/Mfr., bzw. schon weniger richtig, im Sulzbacher Land/Opf. Die Art der Aufmachung, eine Mischung von zum Teil unverstandener Wahrheit mit reiner Erfindung, wobei von der Entdeckung „des ältesten steinzeitlichen Wohn- und Siedlungsplatzes ganz Europas" die Rede war, veranlaßte die Presseagenturen sogleich zu einer Belieferung der wichtigsten Zeitungen des In- und Auslandes mit entsprechenden Nachrichten über die so rasch „berühmt" gewordene Fundstelle. Leider blieb es nicht nur bei dieser unsachlichen Berichterstattung. Vielmehr stellten sich daraufhin am Grabungsort "heimliche" Helfer ein, die bei ihren "Nachforschungen und Überprüfungen" erheblichen Schaden anrichteten und die wissenschaftlichen Grabungen störten. Eine herbe Enttäuschung für den Verfasser, der als alleiniger Entdecker jahrelang in aller Stille, unterstuitzt von wenigen treuen Mitarbeitern, die Untersuchungen durchgeführt hatte! Wissenschaftlichen Kreisen lag bis dahin lediglich ein kurzes Selbstreferat über einen Vortrag vor, welcher anläßlich des III. Internationalen Spelaeologen-Kongresses in Wien gehalten worden war (HELLER 1961). Weitere Vorträge im Rahmen von Fachkolloquien und einigen iagungen hatten hingegen bewußt keinen schriftlichen Niederschlag gefunden.

Nachdem also vorzeitig und, wie schon betont, auf durchaus ungewöhnlichem Wege gewisse Informationen über eine neue quartärgeologisch-paläontologische und vorgeschichtliche Fundstelle an die Offentlichkeit gedrungen sind, sieht sich der Verfasser auf besondere Aufforderung hin selbst zu einer Zusammenfassung der bisherigen Forschungsergebnisse veranlaßt.

Seit dem Einsetzen der erdgeschichtlich-paläontologischen Forschung können die Höhlen des Fränkischen Jura als wahre Schatzkammern der Natur gelten. Waren sie es doch, die schon zu Zeiten eines Johann Friedrich EsPer, sodann eines Johann Christian Rosenmüller und August GoldFuss die Aufmerksamkeit der wissenschaftlichen Welt auf sich lenkten und wesentlich zur Kenntnis der Tierwelt des „Diluviums“ beitrugen. Freilich repräsentierten die „Zoolithen“ der fossilen Großsäuger und die erst viel später beachteten gelegentlichen Anhäufungen von kleineren und kleinsten Wirbeltieren stets nur die Fauna der jüngsten Abschnitte des Quartärs. Erst ab 1927 erfolgte sodann die Entdeckung von Restyorkommen fossilführender Sedimente in fränkischen Höhlen, die dem Pliopleistozän, bzw. dem ältesten Quartär und Altquartär angehören und erneut wichtige Beiträge zur Stratigraphie und Chronologie des Eiszeitalters in Europa lieferten (HELLER 1930a und b, 1933, 1955a und 1963, BRUNNER 1933).

Zweifellos besteht nun aber zwischen diesen beiden Ablagerungskomplexen eine ganz erhebliche zeitliche Diskrepanz, was zu der Auffassung führte, im gesamten fränkischen und darüber hinaus

1) Mit einem Beitrag von K. Brunnacker auf S. 117 dieses Jahrbuches. 
auch im süddeutschen Höhlendiluvium gäbe es keine vollständigeren Schichtenprofile mit älteren als höchstens riß/würm-interglazialen Ablagerungen. (Siehe auch: Heller $1955 \mathrm{~b}$ und $1960 \mathrm{a}$ u. b.)

Diese lange Zeit vertretene These muß nach unseren neuesten Erfahrungen jedoch insofern eine gewisse Modifizierung erfahren, als sie offensichtlich nur für jetzt noch als Höhlen existierende, also jederzeit zugängliche Objekte Gültigkeit hat. Schon rein überlegungsmäßig kann dagegen erwartet werden, daß Höhlenruinen vor allem höher gelegener Verkarstungsniveaus außer den bereits nach. gewiesenen ältest- und altquartären Ablagerungen auch solche des mittleren Pleistozäns beinhalten. Einer Freilegung derselben stehen allerdings in der Praxis insofern beträchtliche Schwierigkeiten im Wege, als man dabei in erster Linie auf das Vorhandensein von Steinbrüchen angewiesen ist, bzw. sein dürfte, die zufällig Teile alter, verfüllter Höhlensysteme anschneiden. Nicht zuletzt bedarf es schließlich auch der Aufmerksamkeit sowie des guten Willens der Steinbruchsleute, allenfallsige paläontologische und auch andere Funde rechtzeitig zu melden und die Bergung dieses Materials zu veranlassen.

Wenn im folgenden nunmehr von Vorkommen vorwürmeiszeitlicher Ablagerungen größeren Umfanges berichtet werden kann, so stellt dies einen unerhörten Glücksfall dar und ist nur dem Umstand zu verdanken, daß der Steinbruchbetrieb zum Erliegen kam, bevor die betreffenden Verfüllungen unbemerkt dem Abbau anheimfielen.

Bei einer systematischen Untersuchung des Gebietes auf hochgelegene verfüllte Karsthohlformen kam Verfasser im Mai 1956 nach jahrelanger Pause erstmals wieder in die an der Ostflanke des Steinberges bei Hunas, bzw. Deinsdorf, befindlichen Dolomitbrüche des Malm $\varepsilon$. Rötliche Färbung des lockeren Gesteinsmaterials in verschiedenen kleineren Spalten und Höhlungen des Hunaser Steinbruches, welcher der Firma Carl Sebald Söhne, Zementwerke in Hartmannshof, Landkreis Hersbruck/Mittelfranken gehört, ließ unter Umständen geologisch ältere faunistische Funde erwarten, und tatsächlich konnten schon sehr bald an einer Stelle einige kleinere Fragmente zweifellos fossiler Knochen und Zähne aufgesammelt werden. Bei einem späteren Besuch des Steinbruches im Herbst 1956 erregten sodann am Fuß einer mächtigen, steilansteigenden Schutthalde zahlreiche frei herumliegende, tiefschwarz gefärbte Knochen meine Aufmerksamkeit. Sie entstammten, wie sich bei näherer Untersuchung ergab, mehreren Fossilhorizonten, welche ungefähr 20 Meter darüber in den Restpartien einer großen, völlig verstürzten Höhle anstanden.

Rund 250-300 cbm Gesteinsmaterial, feinste Dolomitsande, kleinere und größere Gesteinsstücke, gröbere Brocken bis zu riesigen Dolomitblöcken und -platten, wurden seitdem in 7jähriger Grabungsarbeit bewältigt und dabei ein überaus buntes, vielgliederiges, fossilführendes Schichtenprofil freigelegt, das stellenweise eine Mächtigkeit bis zu 10 Meter erlangt, ohne daß bisher die eigentliche Felssohle des ehemaligen Hohlraumes erreicht wurde.

Ganz zu oberst (vgl. Abb. 1 im Beitrag Brunnacker), unmittelbar unter dem Felsdach liegen, zwar nicht überall entwickelt, reine feingeschichtete bräunliche Dolomitsande (A), die keinerlei Fossilreste enthalten. Sie bilden offensichtlich den Abschluß der gesamten Verfüllungen, was schon daraus ersichtlich ist, daß verschiedentlich auf ihnen Teile der eingestürzten Höhlendecke in Gestalt dicker Dolomitfelsplatten aufruhten.

Die nächstfolgenden Profilglieder in ihren tatsächlichen Zusammenhängen und ihrer Verbreitung richtig zu erkennen, fiel vor allem zu Beginn der Ausgrabungsarbeiten nicht immer leicht, da der petrographische Charakter der Sedimente nach Korngröße, Beschaffenheit der gröberen Komponenten (Intensität der Verwitterung, Grad der Verrundung usw.), sowie Färbung zuweilen rasch wechselte und deutliche Abhängigkeit von mindestens 2 ehemaligen größeren Höhleneingängen und verschiedenen Lieferspalten zeigte. Erst beim weiteren Fortgang der Arbeiten wurde immer klarer, daß die Verfüllungsmassen das Material mehrerer, völlig ineinandergeschachtelter Schuttkegel repräsentieren. Zwangsläufig mußten sich somit in den einzelnen Profilschnitten immer neue Bilder ergeben.

Im Normalprofil lagerten unter den geschichteten reinen Dolomitsanden rötlich-braune Dolomitsande mit viel kantigem, zum Teil zersetztem Dolomitschutt (B), sowie bräunlichlehmige Dolomitsande mit zahlreichen festen Dolomitbrocken (C). Beide Schichtenglieder 
enthielten nur spärliche Fossilreste von Höhlenbär, Hirsch, Nashorn usw. Die tieferen gelblich-rötlichen, teils mit eckigen, teils mit auffallend gerundeten Gesteinsbrocken durchsetzten Dolomitsande (D) hingegen lieferten eine reiche Ausbeute an im bergfeuchten $\mathrm{Zu}-$ stande tiefschwarz gefärbten Knochen. Vorwiegend handelte es sich um Reste eines Höhlenbären, sodann um solche von Hirsch, Reh, Biber und Nashorn. Hinzu kommen noch relativ häufig Schneckenschalen von Heliciden.

Deutlich hob sich bei den anfänglich angelegten Längsschnitten eine graue Schicht (E) $a b$, aus der wiederum zahlreiche tiefschwarz gefärbte, später stark aufhellende Knochenreste geborgen werden konnten: sehr häufig Höhlenbär, seltener Hirsch, Reh, Biber, Wildpferd und Wisent. Nahe dem ehemaligen Südeingang, unter dem dort weit vorspringenden Höhlendach, fanden sich außerdem vereinzelt, dennoch auf engerem Raume beieinander liegend, über 50 Silexstücke, meist stark angewitterte Jurahornsteine, ferner 1 karbonischer Kieselschicfer, welche zum Teil deutliche Spuren einer Bearbeitung erkennen lassen. Beim Fortgang der Grabungen zeigte sich, daß die graue Schicht bergwärts immer schwächer wurde und sich zuletzt ganz verlor. Sehr schön konnte bei Querprofilen gelegentlich eine regelrechte Ablösung der grauen durch die darüberliegende Dolomitsandschicht $\mathrm{D}$ beobachtet werden.

Als recht fossilreich erwies sich, wenigstens in ihrem oberen Teil, die folgende, bis zu 1 Meter mächtige gelbgraue Schicht (F). Mit größter Wahrscheinlichkeit darf sie wohl als weniger stark humifizierte untere Partie der grauen Schicht aufgefaßt werden. Das frisch geborgene Knochenmaterial zeigte meist braunrote Färbung und lieferte im großen und ganzen dieselben Arten wie Schicht E.

Mit einer Mächtigkeit von über 1 Meter schloß sich sodann im nordöstlichen Teil der Grabungsstelle ein nahezu fundleerer Komplex grauen bis gelblichen, gelegentlich auch hellbräunlichen Dolomit-Bruchschuttes (G) mit Dolomitsandlagen und einer deutlichen rötlichen $Z_{\text {wischenzone }}\left(\mathrm{G}_{2}\right)$ an. Nahe seiner Basis fanden sich in weiter Verbreitung stark zersetzte Holzkohlen, teils nur einzelne Flitterchen oder Stückchen, hin und wieder kleinere Nester, aber auch ausgedehntere, zu einer Lage von 5-7 cm Dicke zusammengeschlossene Vorkommen. Die recht schwierige Untersuchung verschiedener Proben durch Dr. A. SELMAIER-München ergab stets „Nadelhölzer“, wobei alle wahrnehmbaren Merkmale des Feinbaues auf die Tanne, bzw. auf eine Art der Gattung Abies hindeuten, Durch Feuer gerötete und veränderte Dolomitstücke lieferten ferner den einwandfreien Beweis, daß in der Höhle regelrechte Brandstellen unterhalten worden waren. Kulturelle Hinterlassenschaften des steinzeitlichen Menschen als dem Urheber dieser unübersehbaren Spuren kamen jedoch nur äußerst zögernd und zudem auch überaus spärlich zum Vorschein. Die Grabungen des Jahres 1961 erbrachten trotz stets sorgfältigster Durchsuchung der betreffenden Schichten überhaupt insgesamt nur 4, allerdings unter genauester Beobachtung der Fundumstände gehobene Artefakte aus einem interessanten hellgrauen Kieseloolith, dessen Herkunft vorläufig noch völlig ungeklärt ist. Dieser zwar bescheidene, aber erfreuliche Anfangserfolg berechtigte selbst bei einer gewissen Skepsis zu weiteren Erwartungen.

Unter erstmaliger tatkräftiger Mitwirkung von Herrn Peter SESSLER-Ansbach, dem langjährigen treuen Mitarbeiter des Herrn Dr. K. GuMPERT, ging es daher im Frühjahr 1962 an die Freilegung neuer Querprofilschnitte, welche zunächst die bereits bekannte Schichtenausbildung zeigten. Aus dem unteren Teil der Bruchschuttschicht $\left(\mathrm{G}_{3}\right)$ kamen nur noch 2 größere Abschläge, einer davon abermals aus dem eben erwähnten Kieseloolith, der andere aus gelblichem Jurahornstein, zum Vorschein. Bald aber machte sich eine deutliche

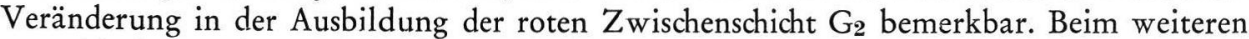
Vortrieb der Grabungen in südwestlicher Richtung und auf einen (?) der früheren Höhleneingänge zu, wurde diese "Zwischenschicht" auf Kosten der $\mathrm{G}_{3}$ mächtiger und dunkler, enthielt stellenweise ein bis zu $10 \mathrm{~cm}$ starkes Kohlebändchen, trat aber auch manchmal in stark versintertem Zustande auf. Ihren Charakter als Kulturschicht bewies sie schließlich 
durch die Herausgabe von insgesamt 180 durchaus ortsfremden Hornsteinstücken, neben Artefakten und kleineren und größeren Retuschensplittern auch etliche rohe Knollen. Dazu gesellten sich reiche Funde an Knochenresten, die wohl zum größten Teil von Jagdbeute des Menschen herrühren dürften. Vorwiegende Formen sind: Höhlenbär, Hirsch, Reh und Elch.

Waren Kleinsäuger- und sonstige Kleinwirbeltierreste in den bisher beschriebenen Schichtgliedern allgemein nur recht spärlich anzutreffen, so stellten sich neuerdings solche in Gesamt-G, hauptsächlich aber in $\mathrm{G}_{2}$ und $\mathrm{G}_{3}$ mit ziemlicher Regelmäßigkeit und zugleich in größeren Anhäufungen ein. Auffallend war das mitunter massenhafte Auftreten von Amphibienknochen. Auch Vogelreste waren nicht selten. Unter den Säugetieren herrschen Vertreter des Microtus arvalis-agrestis-Formenkreises, Microtus ratticeps usw. vor. Für Aussagen in klimatischer Hinsicht ganz besonders bedeutungsvoll ist das, wenn auch spärliche Vorkommen von Dicrostonyx, Cricetiscus und Ochotona (Halsbandlemming, Steppenhamster und Zwergpfeifhase!).

Deutlich hebt sich im Gesamtprofil die nächstfolgende leicht lehmige, graubraune Schicht $\mathrm{H}$ ab. Durch zahlreiche rostbraune Flecken und vereinzelte Kohleflitterchen charakterisiert, enthält sie vielfach von Manganausscheidungen dunkel gefärbte Dolomitstücke, deren mitunter stärkere Verrundung und Vermorschung auffällt. Besonders hervorzuheben ist ferner, daß sich anfänglich kaum nennenswerte Knochenreste fanden und die an und für sich spärlichen Fragmente noch dazu einen überaus starken Grad der Zersetzung aufwiesen. Dies und die eigenartige, beinahe als verknetet zu bezeichnende Struktur legen die Frage nahe, ob es sich hier nicht um eine Ablagerung handeln könne, die längere Zeit freilag, also während einer Sedimentationspause den Höhlenboden bildete, der von Tieren und Menschen begangen wurde (Trampelboden!). Herrn Kollegen Dr. Filzer-Tübingen gelang es, in dieser Schicht Pollen festzustellen, die zu 90\% von Pinus stammen, aber auch Haselnuß, Linde, Eiche, Esche und Erle ausweisen. Während der letztjährigen Grabungen wurde eine Zunahme der durchschnittlichen Mächtigkeit dieser Schicht von bisher $20-25 \mathrm{~cm}$ auf $50 \mathrm{~cm}$, ja sogar $85 \mathrm{~cm}$ beobachtet. Gleichzeitig mehrten sich die Funde besser erhaltener Knochenreste, unter welchen, neben den schon des öfteren genannten Arten, wie Höhlenbär, Hirsch, Reh usw., die Fragmente mehrerer Elchgeweihe Erwähnung verdienen. Menschliche Zutaten aus Schicht $\mathrm{H}$ sind ein Jurahornsteinstück und ein keilförmiges Stück Kalk aus dem Werkkalk des Malm $\beta$, das in einer fast schwarz gefärbten Zone lag. An einer Stelle der graubraunen Schicht wurde eine etwa $7 \mathrm{~cm}$ starke Kohleneinlagerung angeschnitten, die ein Abschlagstück eines größeren Gangquarzgerölles oder -geschiebes lieferte.

Nur eine geringmächtige Lage bilden gelbliche Lehme $(\mathrm{J})$, welche stellenweise überhaupt nicht als eigentliche Schicht in Erscheinung treten, da sie offensichtlich in Lücken und Hohlräume des darunter folgenden, mehr oder weniger verfestigten gröberen Bruchschuttes $(\mathrm{K})$ abgerieselt sind, bzw. verschwemmt wurden, der dadurch gelbliche Färbung angenommen hat. Vielfach mußten daher die mitunter nesterweise vorkommenden Kleinsäuger- und sonstigen Kleinwirbeltierreste, aber auch größere Knochenfragmente usw. zu einer stratigraphischen Einheit $(J+K)$ zusammengefaßt werden. Die Fauna selbst umfaßt, außer kleinen Vögeln, Fledermäuse, Spitzmäuse, Maulwürfe sowie Nager, darunter bemerkenswerterweise Apodemus sylvaticus, womit sich diese Tiergesellschaft mindestens zum Teil als solche des Waldes zu erkennen gibt. Aus dem gelblichen Bruchschutt stammen an größeren Tieren nur vereinzelte Funde von Höhlenbär und Elch (Schädelrest mit Oberkieferbezahnung). Von allergrößter Wichtigkeit sind die Unterkiefer-Fragmente eines kapitalen Wildschweines.

Die in den einzelnen Profilschnitten erschlossenen noch tieferen Verfüllungen scheinen folgende weitere Aufgliederung zu rechtfertigen: ein dunkleres, d. h. humoses Band, unter dem abermals z. T. graugefärbter Bruchschutt (L) kommt, der seinerseits gröberen bis 
groben Versturzblöcken aufliegt, zwischen denen sich als Füllsel gelbliche bis weißliche Dolomitsande finden (2 Meter).

Der zuletzt beschriebene Ablagerungskomplex ist auf Grund der bisherigen Feststellungen nicht gleichartig entwickelt. Im NO der Grabungsstelle besteht nämlich eine durch besonders starkes Einfallen charakterisierte, offensichtlich lokale Sedimentserie unter dem verfestigten Bruchschutt $\mathrm{K}$ aus gelbbraunen, gelblichen und rötlichen Dolomitsanden und Feinschutt, sowie kaffeebraunen, gelbbraunen und ockerbraunen mehr oder weniger lehmigen Ablagerungen. Hier wurde auch im Grenzbereich zu dem groben Bruchschutt an der Basis auf einer Felsplatte eine Feuerstelle von 1,45 x 1,50 m Ausdehnung freigelegt, die in einer solchen Tiefe eigentlich nicht erwartet wurde. Leider konnten außer weitgehend zerfallenen Kohlestückchen weder Knochenreste etwaiger Beutetiere des Menschen noch irgendwelche Artefakte entdeckt werden.

In ihrer Gesamtheit ist die faunistische Ausbeute des Diluvialprofils von Hunas überaus beachtlich. Kaum eine Schicht erwies sich als völlig steril. Die Hauptmasse der Funde entstammt allerdings den rötlichen Dolomitsanden (D), der grauen (E) und der gelbgrauen Schicht $(F)$. Der erst nahezu fundleere Bruchschuttkomplex (G) machte während der Grabungen des Jahres 1962 vieles wieder wett.

Auffällig ist der stark brüchige Zustand der meisten Kiefer- und größeren Knochenreste. Schon in situ weisen diese vielfach deutliche Risse und Sprünge, zuweilen mit regelrechten Versetzungen auf, so daß eine Entnahme aus dem Sediment fast stets nur in Fragmenten erfolgen kann. Gleiches gilt für zahlreiche Zähne, insbesondere für die Eckzähne der Höhlenbären, von denen bisher kaum ein Exemplar beim Bergen völlig intakt blieb. Aber selbst sonst so massive Skelettelemente, wie Phalangen, Metapodien, Carpal- und Tarsalknochen, Patellen usw., zerbrechen mitunter in mehrere Stücke. Die vom Verf. persönlich durchgeführte Konservierung, d. h. das Zusammensetzen des bis heute angefallenen Fossilmaterials nahm allein schon viele Monate in Anspruch.

Häufigste Art der Großfauna von Hunas ist ein Höhlenbär, der hinsichtlich seiner verhältnismäßig geringen Größe und einfachen Zahnskulpturen einen relativ primitiven Eindruck macht. In weitem Abstand, wenn auch durchaus nicht selten, folgen Hirsch und Reh. Weniger häufig finden sich Reste von Elch und Biber, während Wolf, Pferd und Nashorn, Wildschwein und ein Bovide nur in spärlichen Belegen vorliegen. Mit Sicherheit fehlen bisher Höhlenhyäne, Ren und Elefanten. Nennenswerte Mengen von Kleinsäugern (Fledermäuse, Insektenfresser und Nager ), ferner von Vögeln und Amphibien konnten, wie bereits erwähnt, erst im letzten Jahre aufgesammelt werden.

Auf Grund der faunistischen Gegebenheiten, die im Rahmen der Besprechung der einzelnen Schichtglieder ebenfalls schon dargelegt wurden, kann man wohl nur den Schluß ziehen, daß wir es bei den Verfüllungen der ehemaligen Höhle von Hunas in der Hauptsache mit Bildungen zu tun haben, deren Ablagerung vor der letzten Eiszeit anzusetzen ist. Einen wichtigen Fixpunkt bildet hierbei die gut vertretene Fauna der grauen und gelbgrauen Schicht E und F, welche unter allen Umständen nur als zwischeneiszeitlich und zwar riß-würm-interglazial aufgefaßt werden kann. Die hangenden Schichten B/C dagegen verraten durch stärkere Bruchschuttführung zweifellos einen gewissen glazialen Einschlag. Sie scheinen mehr und mehr zur letzten Eiszeit hinüberzuleiten, bzw. bereits den Phasen des Altwürms anzugehören, in dessen Ablauf der weitgehende Einsturz der schon seit langem in Verfall begriffenen Höhle erfolgte.

Konnte sich die Deutung der mächtigen Bruchschuttmassen von $G_{1}-G_{3}$ als glaziale Bildungen bisher lediglich auf den petrographischen Charakter der Ablagerungen stützen, so ist dies mit der Auffindung solch typischer Klimaindikatoren wie Halsbandlemmingen usw. nunmehr zur absoluten Gewißheit geworden. Für die zeitliche Datierung dieses Komplexes, ebenso wie der weiteren sich nach unten anschließenden Teile des Profils kommt dann aber nur eine Zuordnung zur Riß-Eiszeit in Frage. Im einzelnen läßt sich, 
besonders mit Hilfe der in jeder Hinsicht überaus markanten graubraunen Schicht $\mathrm{H}$, eine noch detailliertere Gliederung vornehmen. Ausweislich des oben mitgeteilten Pollendiagrammes dieser Schicht muß deren Bildung während einer zwar etwas wärmeren Klimaphase, keinesfalls jedoch während eines echten Interglazials erfolgt sein. Nichts ist naheliegender als hier die Einwirkungen eines Interstadials innerhalb der rißzeitlichen $\mathrm{Ab}$ lagerungen $\mathrm{zu}$ vermuten. Diesem könnten auch noch die darunterliegenden gelblichen Lehme $(\mathrm{J})$ und ein Teil des gelblichen Bruchschuttes $(\mathrm{K})$ angehören, wohingegen die noch tiefere Abfolge aus mitunter verhältnismäßig grobem Bruchschutt, ja regelrechten Blockanhäufungen, einen älteren Abschnitt der Rißeiszeit repräsentieren dürfte. Ob der zu jener Zeit erstmals sich anbahnende Verfall der ehemaligen Höhle rein klimatische Ursachen hat oder nicht, wird überaus schwer zu entscheiden sein. Eindeutig ging der Hohlraumzerstörung noch eine allenthalben in Resten gut feststellbare starke Versinterung voraus, die man zweckmäßigerweise am besten in das große Mindel-Riß-Interglazial verlegen wird.

Insgesamt gesehen liegt in der Lokalität Hunas ein für das süddeutsche Höhlendiluvium wohl ziemlich einmaliges und ungewöhnliches Schichtenprofil vor, das besonders durch die noch im Gang befindliche Untersuchung der Faunenreste wertvolle Aufschlüsse zur Feinstratigraphie von Zeitabschnitten erwarten läßt, über die, jedenfalls aus unserem Raum, noch recht wenig bekannt ist. Daß bei den geologisch-paläontologisch ausgerichteten Grabungen gleichzeitig auch Hinterlassenschaften des altsteinzeitlichen Menschen in so unerwartet großer Zahl, und zwar in nicht weniger als 5 verschiedenen Niveaus, zum Vorschein kamen, unterstreicht die Bedeutung dieser Fundstelle noch mehr.

\section{Literaturverzeichn is}

BrunNaCKer, K.: Die Sedimente in der Höhlenruine von Hunas (Nördliche Frankenalb). (Vorläufiger Bericht.) - Eiszeitalter u. Gegenwart 13, S. 117-120, Ơhringen/Württ. 1963.

Brunner, G.: Eine präglaziale Fauna aus dem Windloch bei Sackdilling (Oberpfalz). - N. Jb. Min. etc. B, Beil.-Bd. 71, S. 303-328, Stuttgart 1933.

Heller, Fl.: Jüngstpliozäne Knochenfunde in der Moggaster-Höhle (Fränkische Schweiz). - Cbl. Min. etc. B, S. 154-159, Stuttgart 1930 (1930a). - - Eine Forest-Bed-Fauna aus der Sackdillinger Höhle (Oberpfalz). - N. Jb. Min. etc. B, Beil.-Bd. 63, S. 247-298, Stuttgart 1930 (1930b) . - - Ein Nachtrag zur Forest-Bed-Fauna aus der Sackdillinger Höhle (Oberpfalz). - Cbl. Min. etc. B, S. 60-68, Stuttgart 1933. - - Ein kleiner Bär (Ursus sackdillingensis n. sp.) in der cromerischen Fauna der Sackdillinger Höhle (Oberpfalz). N. Jb. Geol. Paläont., Mh., S. 520-530, Stuttgart 1955 (1955a). - - Fauneninhalt und Schichten der Jungfernhöhle. In: Kunkel, O.: Die Jungfernhöhle bei Tiefenellern usw. Münchener Beitr. Vor- u. Frühgesch. 5, S. 52-64, München 1955 (1955b). - - Das Diluvialprofil in der Jungfernhöhle bei Tiefenellern, Landkreis Bamberg. - Erlanger geol. Abh. 34, S. 3-17, Erlangen 1960 (1960a). - - Würmeiszeitliche und letztinterglaziale Faunenreste von Lobsing bei Neustadt/Donau. - Erlanger geol. Abh. 34, S. 19-33, Erlangen 1960 (1960b). - - Ein bedeutsames Profil aus dem fränkischen Höhlendiluvium. - Die Höhle. Z. f. Karst- u. Höhlenkde. 12, S. 92/93, Wien 1961. - - Eine altquartäre Wirbeltierfauna des unteren Cromerium aus der nördlichen Frankenalb. - N. Jb. Geol. Paläont., Abh. 118, S. 1-20, Stuttgart 1963.

Manuskr. eingeg. 18. 2. 1963.

Anschrift des Autors: Prof. Dr. Florian Heller, Nürnberg, Gudrunstraße 26. 